\title{
Diagnosis and outcome in congenital ventricular diverticulum and aneurysm
}

\author{
Eloi Marijon, ${ }^{\mathrm{a}}$ Phalla Ou, MD, ${ }^{a}$ Laurent Fermont, MD, ${ }^{\mathrm{b}}$ Susan Concordet, ${ }^{\mathrm{a}}$ Jérôme Le Bidois, MD, ${ }^{\mathrm{b}}$ Daniel Sidi, MD, PhD, \\ and Damien Bonnet, MD, PhD,
}

From the Department of Pediatric Cardiology, Hôpital Necker-Enfants Malades, ${ }^{\text {a }}$ and the Institut de Puériculture et de Périnatalogie ${ }^{\mathrm{b}}$, Paris, France.

Address for reprints: Phalla Ou, MD, Service de Cardiologie Pédiatrique, Hôpital NeckerEnfants Malades, 149, rue de Sèvres, 75743 Paris Cedex 15, France (E-mail: phalla. ou@nck.ap-hop-paris.fr).

J Thorac Cardiovasc Surg 2006;131:433-7

$0022-5223 / \$ 32.00$

Copyright $\odot 2006$ by The American Association for Thoracic Surgery

doi:10.1016/j.jtcvs.2005.09.046
Objective: True congenital ventricular diverticulum and aneurysm in children are very uncommon. We report our experience to clarify the diagnosis and outcome of these little-known entities.

Methods: Twenty-two patients with congenital ventricular outpouchings were identified in our database from 1973 to 2004. Morphologic characteristics (localization, connection to a ventricle, contractility), histologic findings, and cardiac and/or extracardiac abnormalities were analyzed in all 22 patients. Cardiovascular events and clinical courses were reviewed.

Results: Congenital ventricular diverticula $(\mathrm{n}=16)$ were characterized by synchronal contractility and three myocardial layers on histologic examination. Two categories of congenital ventricular diverticulum could be identified with regard to their localization: apical and nonapical. Apical diverticula $(n=8)$ were always associated with midline thoracoabdominal defects and other heart malformations. Nonapical diverticula $(\mathrm{n}=8)$ were always isolated defects. Congenital ventricular aneurysms $(n=6)$ were characterized by akinesis with paradoxical systolic motion, wide connection to the ventricle, fibrosis on histologic examination that appeared with high signal on T2 weighted magnetic resonance imaging, and absence of other heart or midline thoracoabdominal defects. The outcome was different in these two types of outpouchings: congential ventricular aneurysms were associated with adverse outcomes whereas the prognosis for congenital ventricular diverticula was good.

Conclusion: Congenital ventricular diverticulum and aneurysm are two distinct entities, with different histologic and morphologic characteristics and outcomes. Assessment of these differential characteristics is of importance for prenatal counseling.

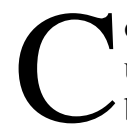

ongenital ventricular outpouchings include congenital ventricular diverticulum (CVD) and aneurysm (CVA). ${ }^{1,2}$ Although various characteristics have been proposed to differentiate the two entities, definition remains incomplete. ${ }^{3}$ In addition, their embryologic origin, clinical profile, natural course, and treatment are unclear since these malformations are rare. Here, we report a single institution experience over a period of 31 years.

\section{Methods}

\section{Patients}

Twenty-two patients with the diagnosis of congenital ventricular outpouching were identified in our department from 1973 to 2004. Demographic characteristics are summarized in Table 1. Medical history, preoperative clinical findings, chest radiography, electrocardiogram, Holter monitoring, prenatal and postnatal echocardiography, magnetic resonance imaging, cardiac catheterization, histologic examinations, and clinical outcome were reviewed whenever available. 


\section{Abbreviations and Acronyms \\ $\mathrm{CVA}=$ congenital ventricular aneurysm \\ $\mathrm{CVD}=$ congenital ventricular diverticulum}

In our series of 22 patients, 16 patients had synchronal contractile ventricular outpouchings and 6 had akinetic or dyskinetic outpouchings. Synchronal contractile outpouchings were defined as congenital ventricular diverticulum (CVD) (Figure 1) and akinetic or dyskinetic as congenital ventricular aneurysm (CVA) (Figure 2).

\section{Statistical Analysis}

All statistical analyses were done with JMP statistical software, version 5.0.1a (SAS Institute Inc, Cary, NC). Data were expressed as the mean $\pm \mathrm{SD}$ and the median with ranges when appropriate. Long-term survivals were calculated by the Kaplan-Meier method and statistical significance was calculated by the log-rank test.

\section{Results}

Characteristics of CVD and CVA are summarized in Table 1.

\section{Morphologic Characteristics}

Sixteen CVDs were identified: 14 concerned the left ventricle and 2, the right ventricle. Two subgroups of CVD could be distinguished regarding their localization in the ventricle. Eight CVDs were localized at the apex and 8 in the other ventricular segments. Apical CVD was a fingerlike contractile pouch with narrow connection to the ventricle

\section{TABLE 1. Population characteristics: CVD and CVA char-} acteristics

\begin{tabular}{|c|c|c|c|}
\hline & \multicolumn{2}{|c|}{ CVD } & \multirow[b]{2}{*}{ CVA } \\
\hline & Apical & Nonapical & \\
\hline \multicolumn{4}{|l|}{ Demography } \\
\hline No. & 8 & 8 & 6 \\
\hline Male/female & $4 / 4$ & $4 / 4$ & $3 / 3$ \\
\hline \multicolumn{4}{|l|}{ Weight at birth $(\mathrm{kg})$} \\
\hline Mean $\pm S D$ & $3.2 \pm 0.2$ & $3.3 \pm 0.2$ & $2.9 \pm 0.3$ \\
\hline Median & 3.25 & 3.2 & 3 \\
\hline Range & $2.8-3.4$ & $3-3.7$ & 2.6-3.3 \\
\hline Age at diagnosis, range & $0 w-8 w$ & $24 w g-8 w$ & $26 w g-16 w$ \\
\hline $\begin{array}{l}\text { Follow-up, mean } \pm S D(y) \\
\text { Other malformations }\end{array}$ & $4.8 \pm 6.5$ & $10.3 \pm 8.4$ & $2.9 \pm 4.4$ \\
\hline Cardiac/midline defects & $7 / 8$ & $0 / 0$ & $0 / 0$ \\
\hline $\begin{array}{l}\text { Mesocardia/dextrocardia } \\
\text { Localization }\end{array}$ & $1 / 6$ & $0 / 0$ & $0 / 0$ \\
\hline $\begin{array}{l}\text { Left ventricle/right } \\
\text { ventricle }\end{array}$ & $7 / 1$ & $7 / 1$ & $4 / 2$ \\
\hline Diagnosis & & & \\
\hline $\begin{array}{l}\text { Prenatal/postnatal } \\
\text { diagnosis }\end{array}$ & $0 / 8$ & $5 / 3$ & $3 / 3$ \\
\hline
\end{tabular}

$S D$, Standard deviation; $w$, weeks; $w g$, weeks of gestation; $y$, years. and was consistently associated with midline thoracoabdominal defects that were visible in 5 patients (epigastric pulsating diverticulum or large omphalocele) and not visible on clinical examination in the 3 others (diaphragmatic dehiscence discovered at surgery that had been indicated for intracardiac abnormalities including tetralogy of Fallot [1 case] and ventricular septal defect [2 cases]). In addition, apical CVD was always associated with cardiac rotation disorder (mesocardia or dextrocardia) and/or intracardiac abnormalities: 3 cases of tetralogy of Fallot, 2 ventricular septal defects, 1 case of ventricle physiology, and 1 case of tricuspid atresia. In contrast, nonapical CVD was a large contractile pouch with wide connection to the ventricle and was never associated with a midline thoracoabdominal defect or other intracardiac abnormalities.

Six CVAs were identified: 4 concerned the left ventricle and 2, the right ventricle. Four CVAs were localized in the anteroapical segment and 2 in the inferobasal ventricular segment. According to the definition, CVA was a large akinetic or dyskinetic pouch. All were characterized by a wide connection to the ventricle. No midline thoracoabdominal defect or congenital heart malformation was observed in patients with CVA. No coronary lesion was described on angiography (3 cases) or histologic examination (3 cases).

\section{Circumstances of Diagnosis}

As mentioned, the 8 apical CVDs were easily diagnosed on postnatal echocardiographic examination because they were associated with other intracardiac malformations and/or midline thoracoabdominal defects. In 5 patients, apical CVD was clinically obvious with an epigastric pulsating diverticulum or large omphalocele. In the other 3 patients, apical CVD was only discovered at surgery as a small diaphragmatic dehiscence.

Five of the 8 nonapical CVDs (62.5\%) were diagnosed on prenatal echocardiography. None of the patients had cardiac dysfunction. The 3 other CVDs were revealed because of benign ventricular extrasystoles during the neonatal period.

Three of the 6 CVAs were diagnosed on prenatal echocardiography, 1 in a patient with normal cardiac function and 2 in patients with left ventricular dysfunction. The 3 others were revealed in the neonatal period by right ventricular rupture, stroke complication, and ventricular extrasystole, respectively.

\section{Treatment and Outcome}

Early and long-term outcome for CVD is shown in Figure 3. By estimated survival analysis, the survivorship for the whole cohort with CVD appeared quite good, with more than $80 \%$ of patients alive at 10 years. Six patients (37.5\%) underwent surgical treatment ( 3 had tetralogy of Fallot, 2 

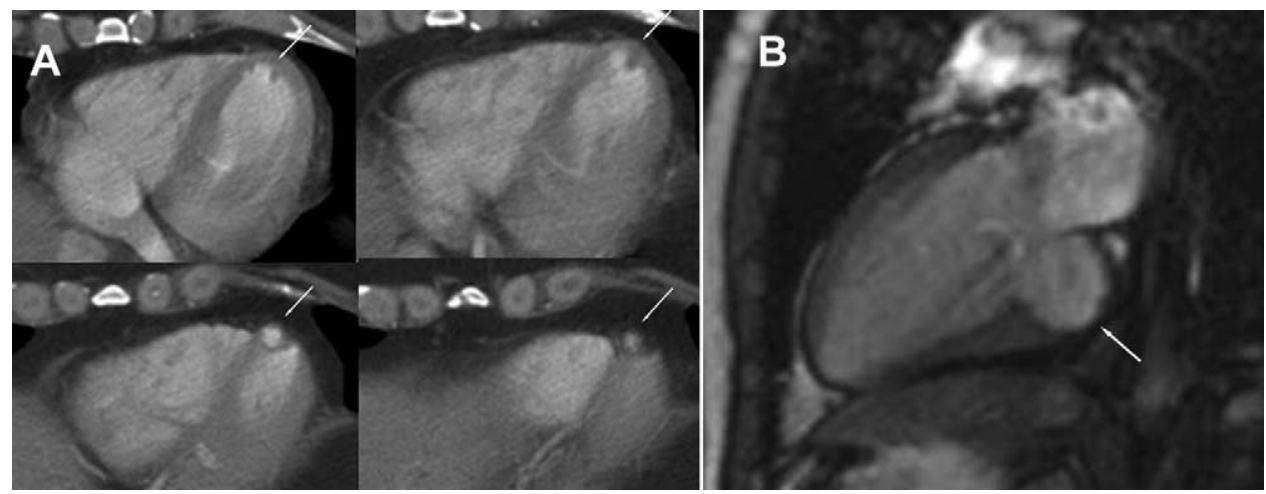

Figure 1. A, Apical CVD of the left ventricle seen on 4-detector-row computed tomography (arrow). B, Nonapical CVD of the inferobasal segment of the left ventricle seen on magnetic resonance imaging (arrow).

had ventricular septal defect, and 1 had tricuspid atresia), whereas the 10 others $(62.5 \%)$ had conservative treatment. Cardiac surgery was never indicated for CVD resection in our series of patients. Two patients died in the early postoperative period due to heart failure (tetralogy of Fallot) and tamponade (tricuspid atresia), whereas the 4 other patients are free of symptoms after a mean postoperative follow-up of 10 years. As anticipated, histologic examination of the 6 surgically treated CVDs confirmed the presence of the 3 myocardial layers without fibrosis. Among 10 patients with conserved CVDs (62.5\%), 2 patients had spontaneous regression of the nonapical CVD and 8 other patients are alive without symptoms after a mean follow-up of 8.4 years.
Early and long-term outcome for CVA is shown in Figure 3. CVA, as compared with CVD, had a dramatically bad prognosis, with only $30 \%$ of patients alive at 4 years $(P<.0001)$. During the first 4 postnatal months, 4 patients with a CVA had severe early complications. Two patients died of heart failure at 1 day of age because of an extremely large aneurysm located in the left ventricle that had been diagnosed on prenatal echocardiography. One patient had tamponade at 3 months of age because of rupture of an aneurysm that was located in the inferobasal segment of the right ventricle. The aneurysm was relatively small, so resection did not lead to any significant impairment of ventricular function. The patient remains free of symptoms 2 years after the operation. One patient died at 4
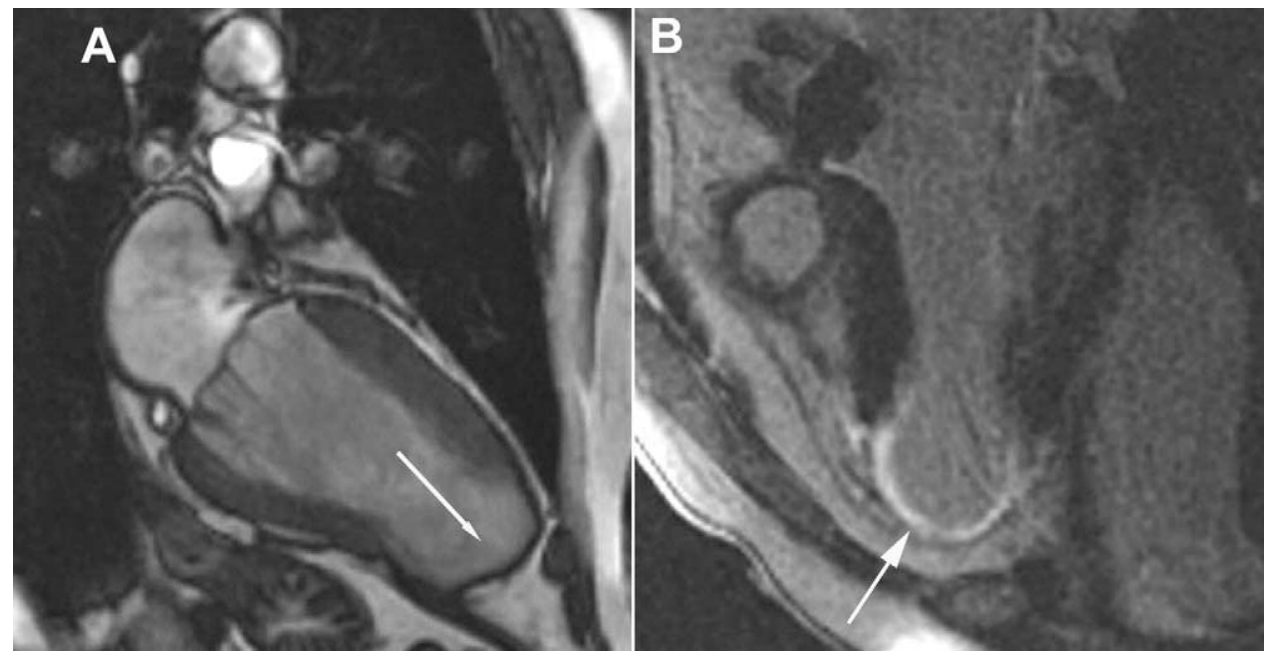

Figure 2. A, CVA on the anteroapical segment of the left ventricle (arrow). B, Delayed contrast enhancement attesting of fibrosis on magnetic resonance imaging (arrow). 


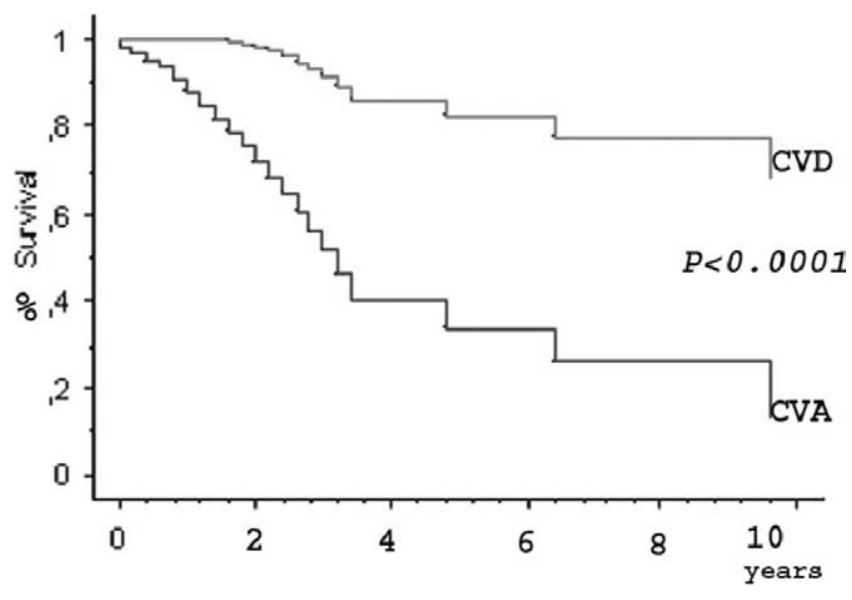

Figure 3. Actuarial curves of long-term survival by the KaplanMeier method.

months of age because of a massive embolic stroke originating from a large aneurysm of the left ventricle. The 2 patients without complications were not operated on, received only anticoagulant therapy, and were still alive at 5 and 11 years, without any evolution in echocardiography. They had uncomplicated ventricular extrasystoles recorded on electrocardiographic Holter monitoring. As previously reported, 4 histologic examinations ( 3 autopsies and $1 \mathrm{op}-$ erated CVA) revealed transmural or endocardial fibrosis.

\section{Discussion}

Our study has confirmed that CVD and CVA are two distinct entities with different morphologic and histologic characteristics and outcomes. The interesting findings in our study include the demonstration of apical CVD as a part of a syndrome of midline thoracoabdominal defect with other intracardiac malformations that had been described by Cantrell, Haller, and Ravitch, ${ }^{4}$ whereas nonapical CVD and CVA were shown as isolated cardiac defects.

\section{Definition}

In the present study, we demonstrated that CVD could be easily differentiated from CVA in daily clinical practice. Objectively, CVD was characterized by synchronous contractility that has been recognized as the most reproducible parameter for diagnosis. ${ }^{5-7}$ Similarly, we found systolic contractility as a constant characteristic of CVD, which was correlated to the presence of muscular fibers within the diverticular wall on histologic examination. This contractile property explains the term muscular $C V D$, frequently used in the literature. ${ }^{7,8}$ Most importantly, we distinguished apical from nonapical CVD. When apical, CVD was a fingerlike contractile pouch with narrow connection to the ventricle. It was a part of the Cantrell syndrome, always including midline thoracoabdominal defects and intracardiac malformations. ${ }^{4}$ When nonapical, CVD was a large contractile pouch with wide connection to the ventricle and was never associated with intracardiac or extracardiac abnormalities.

In contrast, CVA was a large akinetic or dyskinetic pouch with wide connection to the ventricle and endocardial or transmural fibrosis on histologic examination..$^{2-5}$ As previously reported, CVA was never associated with intracardiac or extracardiac defects.

\section{Etiology}

Whereas the etiology of apical CVD is relatively understood, the origin of nonapical CVD and CVA is the subject of various hypotheses. Apical CVD is a part of the Cantrell syndrome ${ }^{4}$ and has a common defect in embryologic development with midline thoracoabdominal formation. The mechanism may result from a failure of normal fusion of the paired primitive mesoderm in combination with abnormal fusion of the cardiac loop to the yolk sac before it descends, leading to the formation of muscular apical CVD. ${ }^{4,7}$ In this condition, a large midline thoracoabdominal defect is generally symptomatic with obvious epigastric pulsatile diverticulum or omphalocele, whereas a small dehiscence is misdiagnosed and discovered only at surgery.

The etiology of nonapical CVD and CVA has been attributed to a focal defect of the muscular ventricular wall due to an intrinsic abnormality in embryogenesis. ${ }^{3}$ CVA has also been suspected to be acquired in the prenatal period, potentially as a result of a viral infection ${ }^{9-11}$ or coronary lesions including stenosis, hypoplasia, and localized intimal proliferation. ${ }^{12}$

CVD and CVA are qualified as congenital in origin when no causes for an acquired defect are discovered. Fetal echocardiography may help to support a congenital etiology when the diagnosis is made during the prenatal period with no evidence of an acquired abnormality. When first detected late in childhood or adulthood, it is more difficult to determine whether an intrathoracic outpouching of the ventricle is congenital or acquired. In this condition, cardiac catheterization and angiography are usually performed to eliminate coronary lesions. ${ }^{1}$ In our study, no evidence of an acquired defect was found.

\section{Diagnosis}

Whereas apical CVD is usually revealed by a midline thoracoabdominal defect and/or other heart malformations, nonapical CVD is more often misdiagnosed, so that it may be more prevalent than previously believed. Indeed, the prevalence of CVD increases as noninvasive methods like fetal echocardiography improve. For example, $62.5 \%$ of nonapical CVDs were diagnosed on prenatal examination in the 10 last years in our department. 
Whereas echocardiography is the method of choice for the diagnosis of congenital ventricular outpouching and other associated cardiac defects during the antenatal ${ }^{9-11}$ and neonatal periods, it becomes more difficult as children grow. In this condition, magnetic resonance imaging is much more useful because it distinguishes CVA fibrosis with delayed contrast enhancement from CVD that has a normal signal (Figure 2) and should therefore be recommended in long-term follow-up. In our study, chest radiography was helpful in the diagnosis of apical CVD when it showed dextrocardia or mesocardia, which is a part of the Cantrell syndrome. Whereas calcification was a classic sign in adult patients having a large $\mathrm{CVA},{ }^{11}$ it was absent in children and young adults in our study. Electrocardiography did not help with the diagnosis of CVD or CVA in our study, sometimes showing nonspecific anomalies. ${ }^{13}$ Holter electrocardiography was more interesting as it recorded ventricular arrhythmias in 5 patients ( 3 nonapical CVDs and 2 CVAs). In our experience, angiography was performed only when acquired etiology was suspected and/or for investigation of complex congenital heart disease associated with apical CVD.

\section{Prognosis and Outcome}

The prognosis for apical CVD depended on associated intracardiac malformations and was generally good after repair. The outcome for nonapical CVD was also excellent, with even a total regression in 2 cases after 22 years of follow-up. In contrast, CVA had a poor prognosis with frequent fatal cardiovascular complications in the neonatal period. In our study, 2 of the 3 patients with a CVA that had been diagnosed during the antenatal period died of heart failure within a few days after birth. Whenever possible, prenatal management should be discussed during parental counseling, especially if CVA is associated with poor prognostic factors on fetal echocardiography, ${ }^{2}$ such as size and progression of the CVA and signs of cardiac failure. ${ }^{11}$

\section{Treatment}

How to treat CVD and CVA remains unresolved. When symptomatic or when associated with other cardiac abnormalities, surgical treatment is usually recommended. Controversy concerns whether an asymptomatic and isolated CVD should be treated surgically. CVD can potentially give rise to embolism, arrhythmias, cardiac failure, and rupture. $^{7,14,15}$ Some authors have proposed resection of all CVDs to prevent these complications, ${ }^{1,7}$ whereas others have adopted conservative management. ${ }^{16}$ We do not recommend surgery systematically because the risk of postoperative cardiac heart failure and death is high; the latter is related to the resection of the diverticular wall, which consists of normal contracting myocardium.
A different approach might be considered for asymptomatic CVA with severe endocardial fibrosis or transmural fibrosis because paradoxical contractions cause dilatation and expose the aneurysm to the risk of rupture. Surgical treatment is usually performed in this condition. Similarly, there is a certain consensus to recommend surgical treatment when CVA is associated with complications. ${ }^{7}$

\section{Conclusion}

CVD and CVA are two distinct entities and should not be confused. They differ by their histologic and morphologic characteristics and outcomes. Assessment of these differential characteristics is of importance for prenatal counseling.

\section{References}

1. Hamaoka K, Onaka M, Tanaka T, Onouchi Z. Congenital ventricular aneurysm and diverticulum in children. Pediatr Cardiol. 1987;8:16975 .

2. Cavalle-Garrido T, Cloutier A, Harder J, Boutin C, Smallhorn JF. Evolution of fetal ventricular aneurysms and diverticula of the heart: an echocardiographic study. Am J Perinatol. 1997;14:393-400.

3. Treistman B, Cooley DA, Lufschanowski R, Leachman RD. Diverticulum or aneurysm of left ventricle. Am J Cardiol. 1973;32:119-23.

4. Cantrell JR, Haller JA, Ravitch MM. A syndrome of congenital defects involving the abdominal wall, sternum, diaphragm, pericardium, and heart. Surg Gynecol Obstet. 1958;107:602-14.

5. Krasemann T, Gehrmann J, Fenge H, Debus V, Loeser H, Vogt J. Ventricular aneurysm or diverticulum? Clinical differential diagnosis. Pediatr Cardiol. 2001;22:409-11.

6. Chesler E, Tucker RB, Barlow JB. Subvalvular and apical left ventricular aneurysms in the Bantu as a source of systemic emboli. Circulation. 1967;35:1156-62.

7. Okereke OU, Cooley DA, Frazier OH. Congenital diverticulum of the ventricle. J Thorac Cardiovasc Surg. 1986;91:208-14.

8. Archbold RA, Robinson NM, Mills PG. Long-term follow-up of a true contractile left ventricular diverticulum. Am J Cardiol. 1999;83:810-3, A11.

9. Lupoglazoff JM, Ricard G, Luton D, Aujard Y, Magnier S, Azancot A. Antenatal diagnosis of congenital left ventricular aneurysm. Arch Mal Coeur Vaiss. 2003;96:529-33.

10. Brachlow A, Sable C, Smith S, Slack M, Martin G. Fetal diagnosis and postnatal follow-up of an asymptomatic congenital left ventricular diverticulum. Pediatr Cardiol. 2002;23:658-60.

11. Bouramoue C, Nkoua JL, Gombet T, Kimbally-Kaky G, Ekoba J. Idiopathic subvalvular aneurysm of the left ventricle. Anatomical, clinical and developmental characteristics. Apropos of 13 cases. Ann Cardiol Angeiol. 1995;44:7-13.

12. Paronetto F, Strauss L. Aneurysm of the left ventricle due to congenital muscle defect in an infant: report of a case with discussion of pathogenesis of associated endocardial fibroelastosis. Am J Cardiol. 1963; 12:721-9.

13. Barboteu M, Desnos M, Hagege A, Dufour M, Chauvaud S, Junes G, et al. Giant negative $\mathrm{T}$ waves in idiopathic apical diverticulum of the left ventricle in adults. Arch Mal Coeur Vaiss. 1995;88:1475-7.

14. Obadia JF, Girard C, Chevalier P, el Farra M, Flamens C, Kirkorian G, et al. Aneurysms of the left ventricle without atheromatous coronary artery disease. Apropos of 2 cases. Arch Mal Coeur Vaiss. 1996; 89:755-9.

15. Suilen C, Friedli B, Rutishauser W. Congenital intrathoracic left ventricular diverticulum in an adult. Chest. 1990;98:750-1.

16. Mardini MK. Congenital diverticulum of the left ventricle: report of two unusual cases. Br Heart J. 1984;51:321-6. 